\title{
Reinhard Keil
}

\section{Gestaltung virtueller Forschungsumgebungen für die philologische Detailarbeit}

\section{Einführung}

Im Bereich der Digital Humanities werden vielfältige Informatikprodukte betrachtet, die von einfachen „inhaltsneutralen technischen Werkzeugen“" über Datenbanken und Informationssysteme sowie virtuelle Infrastrukturen bis hin zu komplexen analytischen Algorithmen reichen. Letztere werden in der Informatik zunehmend unter dem Begriff „Data Science“ zusammengefasst. Der Begriff der Inhaltsneutralität dient dazu, die bloße Umsetzung von Anforderungen von der interdisziplinären Erforschung von technischen Lösungsansätzen zu unterscheiden. Ersteres erfordert Technikkompetenz, letzteres jedoch Forschungskompetenz.

Die Nutzung inhaltsneutraler Werkzeuge begründet demnach keine interdisziplinäre Forschungsarbeit, da die „Neutralität“ die Oberhoheit der Hermeneutik gegenüber den formalen Wissenschaften zu garantieren scheint. Demgegenüber stellen die neuen Methoden aus den Bereichen der Data Science und der Computerlinguistik eine neue Herausforderung dar, da sie, so die Thesen des Verbands Digital Humanities im deutschsprachigen Raum: „das ,Wie“ unserer Praxis um eine empirisch ausgerichtete Epistemologie [ergänzen]. ${ }^{\alpha_{2}}$ Gemäß dieser Sicht begründet genau diese epistemologische Herausforderung die Notwendigkeit der inter- bzw. transdisziplinären Zusammenarbeit, die letztlich zur Prägung der E-Humanities bzw. Digital Humanities geführt hat.

1 Digital Humanities 2020, Thesen des Verbands Digital Humanities im deutsch-sprachigen Raum zur 1. Mitgliederversammlung im Rahmen der Tagung „DHd 2014 Digital Humanities - methodischer Brückenschlag oder ,feindliche Übernahme“? Chancen und Risiken der Begegnung zwischen Geisteswissenschaften und Informatik, Passau 2014, online abrufbar unter http://www.dhd2014.uni-passau.de/ fileadmin/dokumente/projekte/digitalhumanities/DH2020-2014-2-Lang.pdf [Stand: 30.Nov. 2015].

2 Ebd., Abschn. 1.1. 
So sinnfällig und notwendig die Unterscheidung in inhaltsneutrale und epistemologisch herausfordernde digitale Techniken und Systeme auf den ersten Blick sein mag, so problematisch oder zumindest präzisierungswürdig erweist sich bei näherer Betrachtung der Begriff der „inhaltsneutralen technischen Werkzeuge“, denn er unterstellt, dass Inhaltsneutralität ein binäres zeitloses Attribut eines technischen Artefakts als Ganzem zugeschrieben werden kann, und blendet damit sowohl die Aspekte der Genese aus als auch die Tatsache, dass diese Werkezuge meist viele Funktionen beinhalten, von denen nur einer Teilmenge dieses Attribut berechtigterweise zugeschrieben werden kann.

Schon die Nutzung inhaltsneutraler Werkzeuge wie z. B. einer relationalen Datenbank bereitet Probleme, da für Datenbankeinträge zeitlich präzise Angaben erforderlich sind, die im jeweiligen Kontext der Forschung (noch) nicht vorliegen und damit auch nicht als Voraussetzung gesetzt werden dürfen. Allerdings begründet dies noch keine interdisziplinäre Forschungsarbeit, wenn man die Bedingung aufstellt, dass das Werkzeug erst verwendet werden soll, wenn die erforderlichen präzisen Daten vorliegen. Das Datenbankwerkzeug behält seine Inhaltsneutralität, verlagert aber den damit verbundenen Aufwand auf die Forschungsprozesse in den Humanities. Zur Reduzierung dieses Aufwands könnte man jedoch auch interdisziplinäre Projekte nutzen, um neue Ansätze für eine „agile Datenmodellierung“ zu entwickeln, die es gestatten, z. B. das Format, die Granularität und die Verknüpfung von Wissensartefakten im Prozess der Erforschung anzupassen. Werkzeuge zur Unterstützung dieses Prozesses können nicht mehr als inhaltsneutral charakterisiert werden, könnten diesen Status aber partiell erreichen, wenn im Verlauf mehrerer Nutzungs- und Revisionszyklen die Designentscheidungen mit den damit verbundenen Handlungskonsequenzen von der jeweiligen Forschungsgemeinschaft als inhaltsneutral gewertet würden. Der Begriff der Inhaltsneutralität verkörpert somit eher ein Kontinuum als eine binäre Variable und die Platzierung bzw. Zuschreibung einzelner Systeme ist in soziale Zusammenhänge der Genese dieser Werkzeuge eingebettet.

Damit stellt sich für viele zunächst scheinbar inhaltsneutrale Werkzeuge grundsätzlich dieselbe epistemologische Herausforderung wie beim distant reading, wo - analog zum neuen Feld des „,ognitive computing“ - die Inhaltsneutralität nur unter Berücksichtigung vielfältiger Kontextbedingungen und nur in eingeschränkten Bereichen erreicht wird. ${ }^{3}$

Grundsätzlich ist jedoch die Inhaltsneutralität von technischen Verfahren auch aus dem Bereich Data Science ein immanentes Ziel, denn erst eine zumindest graduell

3 Scott Earley, Cognitive computing, analytics, and personalization, in: IT Professional 17 (2015), S. 12-18. 
gesicherte Neutralität gewährleistet, dass die Verfahren übertragbar und ohne spezifische technische Kenntnisse und Kompetenzen nutzbar sind. Allerdings ist hier allgemein anerkannt, dass Inhaltsneutralität ein forschungsmäßig zu erkämpfender Freiraum ist, der nicht per se gegeben ist.

Was für die algorithmische Verarbeitung und Generierung von Wissen gilt, gilt jedoch auch für virtuelle Forschungsumgebungen, die sich die Unterstützung der Wissensarbeit zum Ziel setzen. Dies zeigt sich an verschiedenen Problemen, die eine Herausforderung an die Gestaltung von Unterstützungsfunktionen für die Informatikforschung auch in anderen anwendungsbezogenen Bereichen wie z. B. dem E-Learning darstellen:

- Syntaktische und materielle Strukturen der zu erforschenden Wissensartefakte entsprechen in der Regel nicht semantischen Einheiten der Interpretation.

- Die Granularität der zu bearbeitenden Objekte (Zeichen bzw. Zeichenaggregate) und Strukturen stehen zu Beginn des Forschungsprozesses noch nicht fest bzw. sind im Forschungsprozess selbst Veränderungen unterworfen.

- Hermeneutische Prozesse im Forschungsdiskurs beziehen vielfältige Aspekte ein, die nicht selbst Teil der in Form von Zeichen repräsentierten und zu untersuchenden Gegenstandsbereiche sind.

- Verletzungen der Inhaltsneutralität - auch tradierter analoger Medien - sind nicht im Vorhinein bekannt, sondern müssen erst im Laufe des Forschungsprozesses untersucht bzw. aufgedeckt werden (Wechselwirkung von Materialität und Immaterialität von Zeichen). ${ }^{4}$

- Mehrdeutigkeiten, unvollständiges Wissen sowie verschiedene Varianten und Versionen müssen so kodifiziert werden, dass ihre Anschlussfähigkeit für weitere Fragestellungen und Untersuchungen gegeben ist.

4 Dieser Aspekt, der sich im traditionellen Forschungskontext als zunehmend bedeutsam erweist (siehe Markus Hilgert, ,Text-Anthropologie؛: Die Erforschung von Materialität und Präsenz des Geschriebenen als hermeneutische Strategie, in: Altorientalistik im 21. Jahrhundert. Selbstverständnis, Herausforderungen, Ziele, hg. von Markus Hilgert, Berlin 2010 (Mitteilungen der Deutschen Orient-Gesellschaft 142)), erweist sich bei der Suche „nach der differencia specifica des digitalen im Vergleich zum analogen Dokument“ (Sarah Dudek, Schöne Literatur binär kodiert: Die Veränderung des Text- und Dokumentbegriffs am Beispiel digitaler Belletristik und die neue Rolle von Bibliotheken, Berlin 2011 (Berliner Handreichungen zur Bibliotheks- und Informationswissenschaft 290), S. 11) als Herausforderung, aber erst recht bei der Untersuchung der Wechselwirkungen zwischen beiden. Joachim Veit, Es bleibt nichts, wie es warWechselwirkungen zwischen digitalen und, analogen 'Editionen, in: editio 24 (2010), S. 37-52. 
Inhaltsneutralität ist also keine Kategorie, mit der sich die Relevanz oder Zugehörigkeit von Ansätzen, Methoden und Werkzeugen aus der Informatik zum Feld der Digital Humanities begründen ließe, sondern die Frage, unter welchen Bedingungen und bis zu welchem Grad die Zuschreibung dieses Attributs zulässig ist, stellt selbst den Kern der epistemologischen Herausforderung dar.

Dies beinhaltet auch eine Herausforderung für die Informatikforschung, denn aufgrund der angeführten Besonderheiten ist es nicht möglich, Unterstützungsfunktionen im Rahmen eines benutzerzentrierten Entwicklungsansatzes zu erheben, da die Nutzer weder die innovativen Potenziale der Technik hinsichtlich ihrer zu bewältigenden Probleme abschätzen oder bewerten noch ihre Bedarfe im Vorhinein detailliert artikulieren können. Schon am Beispiel der Entwicklung gebrauchstauglicher Nutzungsschnittstellen ist deutlich geworden, dass die Konzentration auf Nutzer und ihre Aufgaben ein notwendiges, aber keineswegs hinreichendes Merkmal einer ergonomischen Gestaltung ist. ${ }^{5}$

Gewiss ließen sich viele dieser Herausforderungen dadurch ,lösen', dass man nur über genügend lange Jahre mit entsprechendem Aufwand an kompetenten Entwicklern in vielen Zyklen immer wieder die entsprechenden Werkzeuge und Systeme anpasst und weiterentwickelt. Allerdings wäre der Preis für ein solches Vorgehen nach Versuch und Irrtum eine äußerst mangelhafte Kosteneffizienz, da jeder Lernprozess immer wieder teuer bezahlt werden müsste. Zudem wäre es kein taugliches Mittel, um sich im nationalen und internationalen Forschungswettbewerb in den Digital Humanities einen Vorsprung zu erarbeiten oder eine Spitzenposition zu erobern.

Der Ansatz einer hypothesengeleiteten und damit prospektiven Technikgestaltung soll dieses Defizit beheben helfen. Er hat sich bereits bei der Entwicklung von E-Learning-Systemen bewährt und über den nachfolgend skizzierten Begriff der Wissensarbeit können viele der dort angestellten Überlegungen auch auf die Entwicklung virtueller Forschungsumgebungen übertragen werden. ${ }^{6}$

5 Vgl. Reinhard Keil, Hypothesengeleitete Technikgestaltung als Grundlage einer kontextuellen Informatik, in: Informationstechnik und ihre Organisationslücken. Soziale, politische und rechtliche Dimensionen aus der Sicht von Wissenschaft und Praxis, hg. von Andreas Breiter und Martin Wind, Berlin 2011, S. 165184; Reinhard Keil und Christian Schild, Gestaltungskonflikte in der Softwareergonomie, in: Mensch \& Computer 2013: Interaktive Vielfalt, hg. von Susanne Boll, Susanne Maßß und Rainer Malaka, München 2013, S. $67-77$.

6 Jonas Schulte, Reinhard Keil und Andreas Oberhoff, Unterstützung des ko-aktiven Forschungsdiskurses durch Synergien zwischen E-Learning und E-Science, in: Wissensgemeinschaften:Digitale Medien - Offnung und Offenheit in Forschung und Lehre, hg. von Thomas Köhler und Jörg Neumann, Münster 2011, S. 8191. 
Nachfolgend sollen einige grundsätzliche Überlegungen zu den Grundlagen einer hypothesengeleiteten Entwicklung von Unterstützungsfunktionen für die Wissensarbeit skizziert werden. Dabei wird deutlich, dass eine klare Trennung zwischen technischen Funktionen und sozialen Nutzungspraktiken erforderlich ist, um Unterstützungspotenziale verdeutlichen zu können. Ein zentrales Konzept ist hierbei der virtuelle Wissensraum. Auf dieser Basis werden dann Gestaltungsdimensionen für virtuelle Wissensräume identifiziert, die es zum einen erlauben sollen, die Eignung verschiedener Systeme für den jeweiligen Anwendungszweck zu untersuchen, und zum anderen Überlegungen anzustellen, wie die Innenarchitektur solcher virtuellen Räume für einen bestimmten Anwendungszweck gestaltet sein sollte. Diese Innenarchitektur verkörpert keine Systemarchitektur im softwaretechnischen Sinne, sondern ist eine konzeptuelle Brücke, die es gestatten soll, technische Potenziale und konkrete Anforderungen systematisch aufeinander zu beziehen und in technische Lösungen zu überführen.

\section{Wissensarbeit und ihre mediale Unterstützung}

Wie in der Einleitung bereits dargelegt, geht es beim vorliegenden Ansatz nicht um die Modellierung von Wissen, sondern um die Prozesse der Wissensarbeit, in denen vorhandenes Wissen selektiert und angepasst sowie neues Wissen erzeugt wird. ${ }^{7}$ Dabei spielt der Begriff des stillschweigenden oder impliziten Wissens eine entscheidende Rolle. ${ }^{8}$ Obwohl der Begriff Wissensarbeit ursprünglich für die Charakterisierung einer nicht-tayloristischen Form der Arbeit in Organisationen geprägt wurde, bietet er sich gerade durch diese Abgrenzung dazu an, beispielsweise auch auf Lern- und Forschungsprozesse übertragen zu werden. Taylors Buch Grundzüge wissenschaftlicher Betriebsführung, das 1913 in deutscher Sprache erschien, wird als paradigmatisch für den Ansatz gesehen, Arbeitsprozesse in möglichst kleine, sich monoton wiederholende Einheiten zu zerlegen, automatisierbare Anteile zu identifizieren und daraus einen neuen, rationelleren Arbeitsprozess zu gestalten, dem der Mensch sich unterzuordnen hat. ${ }^{9}$

7 Peter F. Drucker, The landmarks of tomorrow, New York 1957.

8 Margit Osterloh, Human resources management and knowledge creation, in: Handbook of knowledge creation, hg. von Ikujiro Nonaka und Ichijo Kazuo, Oxford 2007, S. 158-175.

9 Der Begriff Taylorismus hat sich als Synonym für entmenschlichte Arbeitsteilung festgesetzt, obwohl dies in dieser Form nicht nur Taylor und seinem Werk zugeschrieben werden kann (Walter Hebeisen, F. W. Taylor und der Taylorismus: über das Wirken und die Lehre Taylors und die Kritik am Taylorismus, Zürich 1999). 
Der Begriff der Wissensarbeit entzieht sich qua Definition solchen Zerlegungsprozessen und steht damit ebenso paradigmatisch für geistige Handlungen, bei denen das interpretierende Bewusstsein des Menschen die Voraussetzung für das Gelingen ist. Damit einher geht zugleich das Problem, dass sowohl in Bezug auf die Produktivität als auch die Kontrollierbarkeit von Wissensarbeit dem Management epistemologische Grenzen gesetzt sind. ${ }^{10}$ Dies ist insofern von Bedeutung, als sich damit Wissensarbeitsprozesse gemäß ihrer Definition einer formalen Modellierung entziehen, denn das in den Arbeitsprozessen erst geschaffene Wissen kann im Vorhinein nicht antizipiert und damit auch nicht als Grundlage für die Formulierung von Hypothesen genutzt werden.

Deshalb wird ein Zugang gewählt, bei dem die Unterstützungspotenziale für Wissensarbeit nicht über die Analyse von Wissen und Wissensverarbeitungsprozessen begründet werden, sondern über die Untersuchung der Umgebung, in der Wissensarbeit stattfindet. Diese Perspektive lehnt sich an die Kernaussage von Leroi-Gourhan an, der feststellt, dass in Bezug auf unsere kulturgeschichtliche Entwicklung die Evolution des Geistes im Wesentlichen die der vom Menschen geschaffenen Ausdrucksmittel sei. ${ }^{11}$ Gemäß dieser Sicht bildet die Analyse der Mehrwerte physischer Ausdrucksmittel die Grundlage für die Hypothesenbildung bezüglich der Gestaltung technischer Unterstützungsfunktionen.

Es sind diese medialen Ausdrucksmittel, die es dem Menschen gestatten, über seine biologische Konstitution und seine natürliche Lebenswelt hinausgehend

- mit Hilfe medialer Artefakte die „Umwelt zum Sprechen“ zu bringen und diesen Prozess systematisch zu beobachten (Differenzerfahrung) und

- zeit- und ortsübergreifende gemeinsame Handlungs- und Wahrnehmungsräume zu schaffen (externes Gedächtnis).

In welcher Form Begriffe wie Differenzerfahrung und externes Gedächtnis genutzt werden können, um im Rahmen einer hypothesengeleiteten Technikgestaltung Unterstützungsumgebungen auf der Basis virtueller Wissensräume im Gebiet E-Learning zu entwickeln, ist bereits vielfach in seinen verschiedenen Facetten vorgestellt worden. ${ }^{12}$

10 Osterloh, Human resources management and knowledge creation (wie Anm. 8).

11 André Leroi-Gourhan, Hand und Wort, Frankfurt am Main 1988, S. 262.

12 Dies reicht von grundlegenden Überlegungen zur ko-aktiven (Thorsten Hampel und Reinhard KeilSlawik, sTeam: Structuring Information in a Team - Distributed Knowledge Management in Cooperative Learning Environments, in: ACM Journal of Educational Resources in Computing 1(2) (2002), S. 1-27) und moderierten (Reinhard Keil, Detlef Schubert und Harald Selke, Mobile Schreibtische als neue Form des betreuten virtuellen Lernens, in: Andreas Schwill und Nicolas Apostolopoulos (Hg.), 7. e-Learning 
Da die hier geschilderte Sichtweise auf Wissensarbeit mit ihren Randbedingungen für Lernprozesse in gleichem Maße gilt wie für hermeneutische Forschungsprozesse, scheint es zulässig, die Annahmen und bisherigen Ergebnisse aus dem Bereich E-Learning auf die Gestaltung virtueller Forschungsumgebungen zur Unterstützung hermeneutischer Praxen in den Digital Humanities zu übertragen.

Wissensräume können verkürzt gesagt als MediArenen der Wissensarbeit charakterisiert werden. Das sind virtuelle Handlungs- und Wahrnehmungsräume, in denen mediale Objekte in vielfältigen Formen abgelegt, bearbeitet, angeordnet und ausgewertet werden können. Die virtuelle Forschungsumgebung verkörpert gewissermaßen die Arena, die zum Zeitpunkt der Entwicklung des technischen Systems mit ihren Eigenschaften und Merkmalen spezifiziert werden muss, während sich die verschiedenen medial gestützten Verarbeitungsformen von Wissen erst zur Nutzungszeit basierend auf der Performanz der handelnden Akteure ergeben. Da auch diese Sicht, ihre grundsätzliche Bedeutung ebenso wie ihre theoretische Herleitung, schon an anderen Stellen hinlänglich beschrieben worden ist, ${ }^{13}$ soll nachfolgend ein darauf

Fachtagung Informatik (DeLFI) 2009, S. 175-185 (GI-Edition Lecture Notes in Informatics P-153)) Strukturierung von Wissen, über die Einbeziehung virtueller Labore (Ferdinand Ferber, Thorsten Hampel, Franz-Barthold Gockel, Thorsten Pawlak und Rolf Mahnken, Homogeneous administration of experiments in material science for configuration, monitoring and analysis, in: Proceedings of 13th International Conference on Experimental Mechanics (ICEM13), Alexandroupolis 2007, S. 455-456) und neuer Formen ko-aktiver Visualisierungen und Simulationen (Bernd Eßmann, Thorsten Hampel und Frank Götz, An open architecture for collaborative visualizations in rich media environments, in: Proceedings of the 8th International Conference on Enterprise Information Systems (ICEIS 2006), Paphos 2006, S. 367-374) über digitale Semesterapparate (Daniel Büse und Reinhard Keil, Lernen in und mit virtuellen Räumen Medi@renen als Stätten der Wissensarbeit, in: Dirk Meister, Wilhelm Schäfer und Wilfried Hauenschild (Hg.), Hochschulentwicklung innovativ gestalten: Das Projekt Locomotion an der Universität Paderborn, Bd. 53, Münster u. a. 2010, S. 57-71) und die Einbettung didaktisch motivierter Verwaltungsfunktionen (Alexander Roth, René Sprotte, Daniel Büse und Thorsten Hampel, koaLA - Integrierte Lern- und Arbeitswelten für die Universität 2.0, in: Sigrid Schubert, Johannes Magenheim und Martin Wessner (Hg.), 5. e-Learning Fachtagung Informatik (DeLFI), Bonn 2006, S. 221-232) bis hin zu neuen pädagogischen Szenarien des Umgangs mit Wissensvielfalt (Bettina Blanck und Christiane Schmidt, Erwägungsorientierte Pyramidendiskussionen im virtuellen Wissensraum open sTeam, in: Auf zu neuen Ufern! E-Learning heute und morgen, hg. von Djamshid Tavangarian und Kristin Nölting, Münster 2005, S. 67-76; Reinhard Keil und Christian Schild, Hypothesengeleitete Gestaltung von Benutzungsoberflächen, in: Mensch und Computer 2014, hg. von Michael Koch, Andreas Butz und Johann Schlichter, München 2014, S. 265-274).

13 Siehe Reinhard Keil, Perspektiven der Wissensarbeit im digitalen Zeitalter, in: Digitale Edition zwischen Experiment und Standardisierung, hg. von Peter Stadler und Joachim Veit, Tübingen 2009, S. 9-22 (Beihefte zu editio 31); Reinhard Keil, Das Differenztheater. Koaktive Wissensarbeit als soziale Selbstorganisation, in: Automatismen, hg. von Hannelore Bublitz et al., München 2010, S. 205-229; Schulte/Keil/Oberhoff, Unterstützung des ko-aktiven Forschungsdiskurses (wie Anm. 6); Jonas Schulte, Johann Rybka, Ferdinand Ferber und Reinhard Keil, KoForum - Kooperative Forschungsumgebung für die organisationsübergreifende wissenschaftliche Laborarbeit, in: Wissensgemeinschaften: Digitale Medien - Offnung und Offenheit in Forschung und Lehre, hg. von Thomas Köhler und Jörg Neumann, Münster 2011, S. 92-101; Felix 
aufbauender Schritt vorgestellt werden, der sich damit befasst, wie im Rahmen einer hypothesengeleiteten Gestaltung mit Hilfe des Konzepts der Innenarchitektur solcher MediArenen eine Brücke zwischen der Welt der evolvierenden Anforderungen und den ebenfalls evolvierenden technischen Möglichkeiten geschlagen werden kann und die dabei gewonnenen Erfahrungen kodifiziert aufeinander bezogen und - soweit möglich - aggregiert werden können.

Dabei geht es nicht zuletzt darum, den Grad der Inhaltsneutralität zu bestimmen und so epistemologische Probleme offen zu legen. Dazu ist es jedoch erforderlich, technische Potenziale und Nutzungspotenziale bzw. aktuelle Nutzungsformen zu trennen, um einerseits Missverständnisse und falsche Erwartungen zu reduzieren und andererseits die der Gestaltung zugrunde liegenden Annahmen offen zu legen und so dem weiteren Forschungsdiskurs zugänglich zu machen.

Die sorgfältige Trennung von Diskursbereichen ist aber leider keine gängige Praxis in der Systemgestaltung, da sich viele im interdisziplinären Umfeld genutzte Analysemethoden auf das Zusammenspiel technischer und menschlicher bzw. sozialer Aspekte konzentrieren. ${ }^{14}$ Eine sorgfältige Abgrenzung technischer und nicht-technischer Bereiche erfolgt kaum. Beispielsweise werden nicht nur im Bereich kooperationsunterstützender Systeme Begriffe wie Kommunikation, Kooperation, Koordination etc. sowohl für technische Funktionen als auch für soziale Prozesse gleichermaßen verwandt. ${ }^{15}$ Die Schwierigkeit dabei ist, dass nicht mehr klar unterscheidbar ist, was denn jeweils die Leistung der Technik ist und was durch die spezielle Form der Nutzung technischer Funktionen erreicht wird, also letztlich das Ergebnis menschlicher (Wissens-)Arbeit ist. Dazu ein Beispiel: Wenn eine Person A einer zweiten Person B eine Nachricht mit dem Inhalt übermittelt: „Treffen am Freitag um 14 Uhr am Stadtbrunnen“", dann koordinieren sie ihre Handlungen, nutzen dazu aber keine technische Koordinierungsfunktion, sondern (nur) eine technische Kommunikationsfunktion. Die Bereitstellung technischer Kommunikationsfunktionen besteht darin, einen in der Regel gedächtnislosen Kanal zur Übertragung von Signalen bereitzustellen, dessen entscheidende technische Qualität darin besteht, dass alle für die Kommunikationspartner potenziell wichtigen Unterschiede in der Kodierung einer Nachricht auch technisch übertragen werden und deutlich vom Hintergrundrauschen unterscheidbar

Winkelnkemper und Andreas Oberhoff, WebArena - Räumliche Strukturen für die Lernorte der Zukunft, in: 20 fahre Lernen mit dem World Wide Web - Technik und Bildung im Dialog, hg. von Reinhard Keil und Harald Selke, Paderborn 2015, S. 103-117 (HNI-Verlagsschriftenreihe 330).

14 Ein geradezu paradigmatischer Vertreter ist die Akteur-Netzwerk-Theorie, die den analytischen Blick auf komplexe soziale Formen schärft, aber die Forderung nach Gestaltungstransparenz nicht unterstützt.

15 Stephanie Teufel, Christian Sauter, Thomas Mühlherr und Kurt Bauknecht, Computerunterstützung für die Gruppenarbeit, Bonn 1995. 
sind. Ob und inwiefern diese Signale für die Kommunizierenden sinnfällig sind, ist dabei unerheblich, denn die Übertragungsfunktion soll eine Nachricht weder abwandeln noch verfälschen (Abtasttheorem).

Der Tatbestand der Koordinierung erfolgt in diesem Beispiel über die Interpretation des Inhalts der Nachricht nicht durch die Nutzung einer technischen Koordinierungsfunktion. Technisch wird eine Funktion zur Unterstützung von Kommunikationsaktivitäten genutzt, aber keine Funktion zur Unterstützung von Koordinationsaktivitäten. Letzteres wäre dann gegeben, wenn das technische System zusätzliche Daten mit entsprechenden Auswertungsfunktionen bereitstellen würde, um Ereignisse zu verarbeiten, um damit die Abhängigkeiten zwischen verschiedenen Aktivitäten unterschiedlicher Akteure zu verwalten. Ein solches Ereignis kann z. B. das Ändern eines Dokuments oder die Neuerscheinung einer Publikation sein, was dann dazu führt, dass alle ,Abonnenten' für das jeweilige Ereignis automatisch eine Nachricht erhalten. Ein Ereignis kann unmittelbar durch Handlungen der Nutzer oder durch zeitliche, örtliche oder sonstige Bedingungen ausgelöst werden. Unterstützungsfunktionen verkörpern somit eine Entlastung von physischem und mentalem Aufwand und ermöglichen damit auf direkte oder indirekte Art und Weise neue Differenzerfahrungen, die ohne sie im jeweiligen Praxisumfeld aufgrund des damit verbundenen Aufwands nicht oder nicht effektiv und verlässlich möglich wären.

Die Nutzung technischer Funktionen kann dabei durchaus entgegen der ursprünglichen Zweckbestimmung sein. Ein hoher Grad an Flexibilität in der Nutzung lässt zugleich auf einen hohen Grad an Inhaltsneutralität schließen. Doch lässt sich diese Einsicht konstruktiv umsetzen? Mit seinem Ansatz „unanticipated use“ verfolgt Robinson das Ziel, die Gestaltung kooperationsunterstützender Systeme zu verbessern, indem nicht Arbeitsabläufe, Regeln und Vorschriften in den Vordergrund der Analyse gestellt werden, sondern versucht wird, möglichst generisch genutzte bzw. nutzbare Artefakte (common artefacts) zu identifizieren. ${ }^{16}$ Zwar helfen die mit dem Begriff der common artefacts verbundenen Dimensionen (predictability, peripheral awareness, implicit communication, double level language, and overview) den Blick für Probleme einer möglichst flexiblen und damit nicht präzise vorgeschriebenen Nutzung eines Systems zu schärfen, doch bleiben für eine prospektive bzw. hypothesengeleitete Gestaltung zu viele Probleme offen. ${ }^{17}$ Insbesondere sind die Dimensionen nicht auf

16 Mike Robinson, Design for unanticipated use, in: Proceedings of the Third European Conference on Computer Supported Cooperative Work (ECSCW), hg. von Giorgio de Michelis, Carla Simone und Kjeld Schmidt, Dordrecht 1993, S. 187-202.

17 Samuli Pekkola, Designed for unanticipated use: common artefacts as design principle for CSCW applications, in: Proceedings of the Group 2003 International ACM SIGGROUP Conference on Supporting Group Work, New York 2003, S. 359-368. 
Eigenschaften und Merkmale des zu gestaltenden Systems bezogen, sondern auf Nutzungsqualitäten, die schon innerhalb einer relativ homogenen Nutzergruppe stark variieren können. Hinzu kommt, dass der Begriff des common artefact auf die gegenständliche, nicht-digitale Welt bezogen war; schon die Dimension der Erwartbarkeit (predictability) gibt Anlass für vielfältige Gestaltungskonflikte ${ }^{18}$ und stellt bei der Gestaltung digitaler Medien eher eine Herausforderung als einen Lösungsansatz dar. ${ }^{19}$

Gleichwohl ist die mit dem Ansatz verbundene Perspektive verwandt mit der hier vorgestellten Sicht, statt der Prozesse der Wissensarbeit die dabei genutzten Artefakte in den Vordergrund zu stellen. Der gemeinsame Handlungs- und Wahrnehmungsraum kann als common artefact betrachtet werden, der beispielsweise die Dimensionen Gewärtigkeit (peripheral awareness), implizite Kommunikation (implicit communication) und Übersicht (overview) in den Fokus rückt. Die besondere Schwierigkeit besteht jedoch darin, dass die semiotischen Objekte in einem virtuellen Wissensraum nicht mehr die Vertrautheit und Stabilität eines gegenständlichen Raums aufweisen, denn sie

- werden von u. U. vielen Personen während der Nutzung angelegt, verändert oder auch entfernt,

- unterliegen keinen natürlichen Kräften und kaum vertrauten Wirkungen wie z. B. der Schwerkraft, die die Möglichkeiten ihres Arrangements einschränken und dadurch zugleich die Verständnisbildung erleichtern, ${ }^{20}$

- sind algorithmisch auswertbar und

- können sowohl manuell als auch automatisch auf vielfältige Weise miteinander verknüpft werden (Indexierung, Referenzierung, Verknüpfung, Annotierung ...),

- weisen eine oftmals erstaunlich variable Granularität auf, sodass syntaktische Einheiten und Strukturen nicht direkt auf semantische Strukturen abgebildet werden können.

18 Pekkola, Designed for unanticipated use (wie Anm. 17), S. 366.

19 Zum Konzept der Gestaltungskonflikte siehe Keil/Schild, Gestaltungskonflikte in der Softwareergonomie (wie Anm. 5) und Keil/Schild, Hypothesengeleitete Gestaltung von Benutzungsoberflächen (wie Anm. 12).

20 Vgl. hierzu das psychologische Konzept der Affordanz: James J. Gibson, The ecological approach to visual perception, Boston 1979; Harold S. Jenkins, Gibson’s „Affordances“: Evolution of a pivotal concept, in: fournal of Scientific Psychology 12 (2008), S. 34-45; Don Norman, The design of everyday things, New York 1988. 
Insbesondere der letzte Punkt verweist auf ein Problem, das sich schon in der gegenständlichen Welt stellt, aber in der digitalen Welt noch deutlich verschärft wird. ${ }^{21}$ Die Konsequenz ist, dass sich der Artefaktbegriff aufzulösen scheint bzw. je nach Situation neu rekonstituiert werden muss. Insbesondere erfordert die Entwicklung von Unterstützungsfunktionen, sowohl die Granularität der betrachteten Objekte als auch die verschiedensten Möglichkeiten ihrer Verknüpfung detailliert zu betrachten und jeweils auf Anforderungen an die technische Gestaltung zu beziehen, denn nur so können neue Formen der Differenzerfahrung eröffnet, Möglichkeiten einer flexiblen, nicht vorhergesehenen Nutzung ${ }^{22}$ erschlossen, Medienbrüche reduziert, Designkonflikte austariert und Entlastungen von unnützen körperlichen und mentalen Aufgaben umgesetzt werden.

Im Rahmen virtueller Forschungsumgebungen zielt die Entwicklung von Unterstützungsfunktionen für die Wissensarbeit darauf ab, möglichst inhaltsneutrale Werkzeuge für die philologische Arbeit zu entwickeln und dabei die situativen Anforderungen der Nutzer so in eine Innenarchitektur virtueller Wissensräume zu integrieren, dass die entstehenden Systeme zu vorhandenen Praktiken anschlussfähig und zugleich für die langfristige Erhaltung und spätere Bearbeitung geeignet sind. Eine grundlegende Hypothese besagt dabei, dass jeder technisch-medial vermittelten Möglichkeit für neue Differenzerfahrungen Unterstützungspotenzial zukommt. Eine weitere geht davon aus, dass jede Reduzierung erzwungener Sequenzialität das Unterstützungspotenzial vergrößert. Erzwungene Sequenzialiät bedeutet dabei, dass Handlungen, die nur aus technischen Gründen erforderlich sind, aber nicht zur Erreichung des jeweiligen Handlungsziels beitragen, ebenso zu vermeiden sind wie aufgenötigte Handlungsfolgen. Wann jeweils Zusatzhandlungen oder aufgenötigte Folgen vorliegen, kann dabei partiell inhaltsneutral ermittelt werden über die Identifikation von Medienbrüchen oder die Berücksichtigung wissenschaftlicher Erkenntnisse z. B. aus der Psychologie. Hinzu kommen aber viele weitere Gestaltungsaspekte wie methodisch zu entwickelnde inhaltsbezogene Funktionen, die ergonomische Gestaltung, die Verwendung von Datenformaten, die Entwicklung von Metadaten, die Auflösung von Zielkonflikten zwischen verschiedenen Nutzergruppen und das Austarieren von Designkonflikten, bei denen einzelne Anforderungen nur auf Kosten anderer, gleichermaßen berechtigter Anforderungen umgesetzt werden können. Dies ist nicht in

21 Vgl. hierzu die Problematik, den Begriff des Dokuments klar zu definieren: Michael Buckland, What is $a$,document"?, in: Journal of the American Society for Information Science 48 (1997), S. 804-809 und Michael Buckland, What is a „digital document"?, in: Document Numérique 2 (1998), S. 221-230.

22 Flexible Nutzungsmöglichkeiten werden heute insbesondere bei der Suche nach Inhalten mit dem Begriff Serendipity belegt, wenn zuvor nicht erwartete Ergebnisse erzielt werden. Beispielsweise kann die Google Bildersuche Dokumente liefern, die man über eine textuelle Eingabe vielleicht nicht erreicht hätte. 
einem einmaligen Entwicklungsprozess zu leisten. Umso mehr kommt es darauf an, Modelle und Konzepte für eine hypothesengeleitete Technikgestaltung zu entwickeln, um bereits erworbenes Gestaltungswissen festzuhalten, dieses mit neuem Wissen und veränderten technischen Möglichkeiten in Beziehung zu setzen und Konsequenzen abzuschätzen. Mit dem nachfolgend vorgestellten Gestaltungskonzept für die Innenarchitektur virtueller Wissensräume soll dazu ein Baustein für Aspekte der verteilten Nutzung vorgestellt werden.

\section{Innenarchitektur virtueller Wissensräume}

Ziel einer hypothesengeleiteten Gestaltung ist es, eine Brücke zwischen der Welt der Nutzung und den formalen Strukturen der Daten, Algorithmen und Systemarchitekturen zu schlagen. Im Gegensatz zu einer einmaligen, auf speziellen Anforderungen basierenden Systementwicklung geht es darum, Konzepte zu entwickeln, die es gestatten, Annahmen bei der Systemgestaltung offen zu legen und zu überprüfen, das entstandene Wissen in einer expliziten Form dergestalt zu kodifizieren, dass es einerseits weitergegeben werden kann und andererseits Ausgangspunkt für das Bilden neuer Gestaltungshypothesen ist, die wiederum in weiteren Entwicklungsschritten überprüft werden können (vgl. Abb. 1).

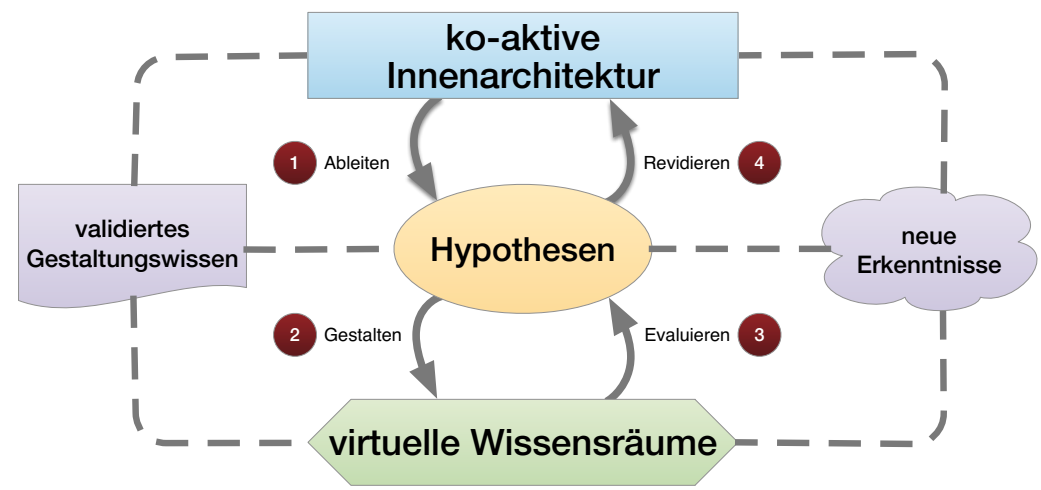

Abbildung 1: Prozess der hypothesengeleiteten Technikgestaltung in Anlehnung an Keil/Schild, Hypothesengeleitete Gestaltung von Benutzungsoberflächen (wie Anm. 12)

Die Theoriebildung zur Entwicklung und Nutzung von Gestaltungswissen bei der Entwicklung von Softwaresystemen ist jedoch über sehr unterschiedlichste Disziplinen 
und Anwendungsfelder gestreut und umfasst neben empirischen Untersuchungskonzepten zur Analyse allgemeiner sozialer Praktiken ${ }^{23}$ kritische Reflexionen zum Gestaltungsprozess selbst ${ }^{24}$ methodische Ansätze zur Verknüpfung von Analyse und Gestaltung und Ansätze für eine theoriegeleitete Anwendungsentwicklung. ${ }^{25}$ Diese bieten zwar vielfältige Anknüpfungspunkte zu den hier vorgestellten Problembereichen, doch zielen sie weniger auf eine hypothesengeleitete Gestaltung von Informatikprodukten als vielmehr auf verbesserte analytisch-empirische Vorgehensweisen und die Theoriebildung in anderen Disziplinen auch dann, wenn interdisziplinär verbindende Wissensobjekte im Vordergrund stehen. ${ }^{26}$ Im Hinblick auf eine technische Fokussierung stellt sich beim Objektbegriff dasselbe Problem wie schon beim Dokumentenbegriff und zunehmend auch bei anderen Begriffen wie z. B. dem des IT artefacts: Die Begriffe sind nicht klar zu definieren oder abzugrenzen, wandeln sich sehr schnell und sie offenbaren eine Fülle von verschiedenen Nutzungsmöglichkeiten und semantischen Konnotationen, die ihre Operationalisierung im Kontext von Gestaltungsprozessen unmöglich machen bzw. mit viel Willkür unterlegen.$^{27}$ Doch selbst wenn diese Konzepte punktuell im Rahmen einer soziotechnischen Systementwicklung erfolgreich eingesetzt werden ${ }^{28}$ ermöglichen sie noch keine Theoriebildung aus der Informatik heraus, da sie auf die Theorie- und Konzeptbildung der jeweiligen Ausgangsdisziplin wie z. B. der Soziologie bezogen sind. ${ }^{29}$ Die geforderte klare Trennung zwischen technischen Potenzialen und sozialen Nutzungspraktiken kön-

23 Volker Wulf, Theorien sozialer Praktiken zur Fundierung der Wirtschaftsinformatik. Eine forschungsprogrammatische Perspektive, in: Wissenschaftstheorie und gestaltungsorientierte Wirtschaftsinformatik, hg. von Jörg Becker, Helmut Krcmar und Björn Niehaves, Heidelberg 2009, S. 211-224.

24 Donald A. Schön, The reflective practitioner. How professionals think in action, New York 1983.

25 Peter Fettke, Constantin Houy und Peter Loos, Zur Bedeutung von Gestaltungswissen für die gestaltungsorientierte Wirtschaftsinformatik. Konzeptionelle Grundlagen, Anwendungsbeispiel und Implikationen, in: Wirtschaftsinformatik 52 (1993), S. 339-352 und Alan R. Hevner, Salvatore T. March, Jinsoo Park und Sudha Ram, Design science in information systems research, in: MIS Quarterly 28 (2004), S. 75-105.

26 Das Konzept der boundary objects (vgl. Susan Leigh Star und James R. Griesemer, Institutional ecology, ,translations 'and boundary objects: Amateurs and professionals in Berkeley's Museum of Vertebrate Zoology, in: Social Studies of Science 19 (1989), S. 387-420) ist ein analytisches Konzept zur Theoriebildung in den Sozialwissenschaften, hat aber bis heute das erhoffte Potenzial für eine hypothesengeleitete Gestaltung noch nicht erfüllen können.

27 Vgl. Steven Alter, The concept of ,IT artifact ' has outlived its usefulness and should be retired now, in: Information System fournal 25 (2015), S. 47-60.

28 Vgl. dazu Wolfgang Reinhardt, Awareness support for knowledge workers in research networks. Dissertation, Maastricht 2012 (Dutch Research School for Information and Knowledge Systems 2012-06), URL: http://hdl.handle.net/1820/4193 [Stand: 30. Nov. 2015].

29 Als exemplarisch kann hier der Aufsatz von Riklef Rambow und Rainer Bromme, Was Schöns „reflective practitioner" durch die Kommunikation mit Laien lernen könnte, in: Wissen - Können - Reflexion. Ausgewählte Verhältnisbestimmungen, hg. von Hans Georg Neuweg, Innsbruck 2000, S. 201-219 betrachtet werden, in dem das bereits referenzierte soziologische Konzept des „reflective practitioner“ von D. A. Schön unter einem empirisch-psychologischen Gesichtspunkt kritisch durchleuchtet wird. 
nen sie entsprechend nicht leisten. Das erschwert die Kodifizierung technischen Gestaltungswissens und seine Fortschreibung im Rahmen einer hypothesengeleiteten Gestaltung.

Um die ko-aktive Innenarchitektur virtueller Wissensräume zu beschreiben, müssen die einzelnen Dimensionen der Gestaltung festgelegt und später dann auf Architekturelemente abgebildet werden. ${ }^{30}$ Dieser Ansatz ist im Rahmen der Lehrveranstaltung zur "Gestaltung kooperationsunterstützender Systeme“ in Zusammenarbeit mit Jonas Schulte entstanden. Das Ziel war dabei, den Studierenden ein Hilfsmittel an die Hand zu geben, um verschiedene Formen ko-aktiven Schreibens wie z. B. „shared editing, shared publishing, shared content oder social networks" in Beziehung zu setzen mit bestimmten technischen Systemen wie Wikis, Blogs, Content Management Systemen oder auch Versionsmanagementsystemen, um herauszufinden, ob es für bestimmte Aufgabenkonstellationen gewissermaßen prototypische Werkzeuge gibt und durch welche Qualitäten sie charakterisiert werden können. Ausgangspunkt war die Feststellung, dass bei Systemvergleichen traditionell Features und Funktionen aufgelistet und verglichen werden. ${ }^{31}$ Dabei geht verloren, dass viele Systeme einen vergleichbar komplexen Funktionsumfang haben, sich jedoch von ihrer Entstehung her in ihrem grundlegenden Verarbeitungsparadigma unterscheiden. Dies lässt sich gut am Beispiel von Textverarbeitungssystemen und „desktop publishing“-Systemen illustrieren: Steht beim Textverarbeitungssystem eine beliebig lange Zeichenkette, in die Formatierungs- und Auswertungszeichen eingestreut werden, im Vordergrund der Verarbeitung, so werden beim desktop publishing grundsätzlich Rahmen bzw. Flächen festgelegt, die untereinander zu einem Textfluss verknüpft werden können. Rein formal betrachtet kann prinzipiell mit beiden Systemparadigmen dasselbe Ergebnis erzeugt werden, doch liegt der Unterschied darin, dass die beiden Arten verschiedene Aufgabentypen jeweils unterschiedlich gut unterstützen.

Entsprechend lautet eine grundlegende Gestaltungshypothese, dass das jeweilige technische Implementierungsparadigma bestimmt, in welchen Situationen und bei welchen Aufgaben die Nutzer jeweils einen vermehrten Aufwand haben, weil das Paradigma nicht mit ihrem typischen Aufgabenkontext übereinstimmt. Unter solchen Gesichtspunkten könnte man auch überlegen, wann beispielsweise für die Verwaltung von Daten der Einsatz einer Tabellenkalkulation ausreichend wäre oder wann man auf

30 Eine solche Abbildung wird exemplarisch in Jonas Schulte, Reinhard Keil, Dominik Klaholt und Jannis Sauer, KOI school - towards the next level of communication, organization and integration in education, in: Proceeding of ICEIS $2011-13^{\text {th }}$ International Conference on Enterprise Information Systems 4, Beijing, China 2011, S. 43-52 vorgestellt.

31 Vgl. dazu die kritische Übersicht zu Lernplattformvergleichen in Harald Selke, Sekundäre Medienfunktionen für die Konzeption von Lernlattformen für die Präsenzlehre. Dissertation, Paderborn 2008, URN: urn:nbn:de:hbz:466-20100226011, Kap. 2. 
eine relationale Datenbank zurückgreifen sollte. Gewiss ist ein Implementierungsparadigma nur ein Faktor in der gesamten Systemgestaltung, doch einer, den Nutzer meist nicht explizit oder gar falsch formulieren, sodass er zu ihren sonstigen Anforderungen im Widerspruch stehen kann. Das Einbringen der Entwicklerkompetenz und die Formulierung von Gestaltungshypothesen sind hier unverzichtbar. Für eine partizipative Entwicklung ist die Trennung der Diskursbereiche eine wichtige Zusatzforderung, um Missverständnissen vorzubeugen, denn Anforderungen der Nutzer und Angebote der Entwickler können, müssen sich aber nicht decken. ${ }^{32}$

In heutigen verteilten Systemen geht es in der Regel nicht mehr um ein einzelnes isolierbares Implementierungsparadigma, sondern um das Zusammenspiel einer Vielzahl einzelner Funktionen, die auf unterschiedliche Systeme oder Komponenten verteilt sein können. Um diesem Umstand gerecht zu werden, müssen verschiedene Gestaltungsdimensionen berücksichtigt werden. Die Identifizierung einer Dimension geschieht dabei analog zu dem schon beschriebenen Beispiel der Koordinierungsunterstützung, das hier zur Begründung des Prinzips um zwei weitere Dimensionen ergänzt werden soll. Ein wichtiger Aspekt ist dabei auch der Aspekt der Inhaltsneutralität, weil sie zugleich die Grenze markiert zwischen technischen Leistungen und solchen, die sich durch die spezielle Einbettung in den (sozialen) Nutzungskontext ergeben.

Das erste Beispiel konzentriert sich auf den Begriff der Anmerkung, mit dem ein vielfältiges Geflecht verschiedenster Ansätze, Interessen und Vorgehensweisen verbunden ist, das von algorithmisch generierten Daten, über individuelle Kommentare bis hin zu Indexierungen und Bewertungen reichen kann. Entscheidend ist, welche Art der Nutzung bzw. welche Formen der Auswertung damit verbunden sein sollen. Beispielsweise kann ein Kommentar an einem Text eine Wertung darstellen, ohne dass dies irgendwelche Auswirkungen auf das weitere Systemverhalten hat; d. h. der Kommentar verändert auch nicht das kommentierte Objekt. Änderungen aufgrund dieses Kommentars erfolgen durch Aktivitäten der Nutzer, die ihn interpretieren und gegebenenfalls daraus Konsequenzen ziehen. Der wesentliche Vorteil von Kommentaren ist, dass sie nicht über einen separaten Kommunikationskanal erfolgen, sondern direkt am zu kommentierenden Objekt. Das erspart den Nutzern den Aufwand des Adressierens einer Nachricht an den oder die Autoren und eine umständliche verbale Beschreibung, um welches Objekt es sich handelt und wo bzw. wie man es finden kann. Insofern eröffnet die Möglichkeit des Kommentars eine neue Gestaltungsdimension, weil das System die Daten für eine solche Zuordnung explizit erfassen und verwalten

32 Deshalb sollte die Systemspezifikation, die ja einen Lösungsvorschlag für die Anforderungen der Nutzer verkörpert, auch eine Bewertung enthalten, inwieweit sie den Anforderungen gerecht wird (Christiane Floyd und Reinhard Keil, Adapting Software Development for Systems Design With the User, in: Systems Design For, With, and By the User, Amsterdam u. a. 1983, S. 143 f.). 
muss. Beziehen sich diese Daten nur auf den topologischen Ort der Handlung in einem Wahrnehmungsraum unabhängig von der Syntax oder Semantik des kommentierten Objekts, dann kann man von einer inhaltsneutralen Gestaltung sprechen. Sobald aber der Ort über syntaktische Kategorien adressiert wird, verstärkt sich die Inhaltsabhängigkeit. Erst die Abgrenzung bzw. Schaffung einer neuen Gestaltungsdimension ermöglicht auch zusätzliche inhaltsneutrale Auswertungsfunktionen, die beispielsweise festlegen, wie viele Kommentare sich auf ein Objekt beziehen können, wie sie dargestellt werden, ob man sie filtern kann, usw. Alle diese Funktionen ändern weder das kommentierte Objekt noch sein Verhalten im System.

Sobald man jedoch eine Auswertungsfunktion haben will, die beispielsweise die Anordnung der Objekte aufgrund von Benutzerbewertungen beeinflussen soll (ranking), benötigt man eine weitere Gestaltungsdimension, die jetzt aber einen zusätzlichen, auf den Inhalt bezogenen Aspekt verkörpert. Zwar ist der Inhalt noch immer unverändert, doch wird er jetzt vom System aufgrund der Kommentare in einem anderen Kontext dargeboten. Dadurch kann es passieren, dass schlecht bewertete Inhalte nicht mehr im Wahrnehmungsfeld der Nutzer erscheinen oder nur durch zusätzliche Navigationshandlungen ins Wahrnehmungsfeld gebracht werden können. Ebenso wie Berechtigungen können somit Bewertungen zu einer Segmentierung des Handlungsund Wahrnehmungsraums führen. Daran schließen sich weitere Fragen an, die sich z. B. auf die Frage der individuellen Erschließung (Indexieren, Tagging) oder z. B. des Setzens von Verweisen (Referenzieren) beziehen. Erst wenn diese auch eigene Gestaltungsdimensionen verkörpern, ist es möglich, beispielsweise Schreibrechte unabhängig voneinander für die verschiedenen Gestaltungsdimensionen zu vergeben. So kann jemandem das Recht zum Indexieren, aber nicht zum Verweisen eingeräumt werden oder man kann überlegen, ob nicht auch Verweise (links) kommentiert oder bewertet werden sollen.

Umgekehrt lohnt es sich auch zu überlegen, ob einzelne Gestaltungsdimensionen nicht zusammengefasst werden können, denn jede zusätzliche Gestaltungsdimension erweitert die Möglichkeiten, den ko-aktiven Handlungs- und Wahrnehmungsraum entsprechend zu konfigurieren und dadurch sowohl neue Differenzerfahrungen zu ermöglichen als auch verschiedenste Möglichkeiten zur verteilten Nutzung zu eröffnen. Damit verbunden steigt jedoch auch die Komplexität der Umsetzung und der Lernaufwand für die Nutzung. So kann man z. B. die Dimensionen Bewerten und Indexieren zusammenfassen, wenn die Auswertungsfunktionen des Bewertens keine Ergebnisse ermöglichen, die nicht auch durch geschickt gewählte Datenformate beim Indexieren erreicht werden könnten.

Gestaltungsdimensionen verkörpern Gestaltungspotenziale, um bestimmte Nutzungsformen zu ermöglichen. Im aktuellen Nutzungskontext ist dann zu entscheiden, ob 
bestimmte Funktionen öffentlich oder nur für eine Benutzergruppe oder eine einzelne Person zugänglich sind. Ein weiterer, eng damit zusammenhängender Aspekt ist die Frage, auf welche Bereiche des Inhalts die Sichtbarkeit oder Zugreifbarkeit bezogen werden kann. Dies lässt sich gut am Beispiel des World Wide Web illustrieren. Hier sind die Verweise definitionsgemäß integraler Bestandteil des Inhalts, von dem aus auf andere Seiten bzw. Inhalte verwiesen wird. Soll eine andere Person als der Autor des Inhalts in die Lage versetzt werden, eigene Verweise anzubringen, dann geht das nur, wenn er damit die Möglichkeit erhält, zugleich den gesamten Inhalt zu verändern. Solange Verweise also nicht als getrennte Objekte verwaltet und damit auch mit eigenen, vom jeweiligen Inhalt unabhängigen Berechtigungen versehen werden können, bilden sie keine eigenständige Gestaltungsdimension. Berechtigen selbst ist natürlich auch wiederum als Gestaltungsdimension zu betrachten, denn Rechte müssen vom System erfasst, verwaltet und überprüft werden.

In diesem Sinne verweist der Begriff ko-aktiv auf die technische Möglichkeit, bestimmte Daten verteilt von verschiedenen Personen oder Gruppen bearbeiten zu lassen (Einschreibungen). Ob dieser Vorgang in der jeweiligen Nutzergruppe egalitär oder hierarchisch gestaltet wird, sollte nicht vom technischen System erzwungen, sondern sozial ausgehandelt werden. Dies gilt gleichfalls für die Entscheidung, welche Daten Nutzer oder Nutzergruppen privat oder öffentlich verwalten und ob und wann sie jeweils diese Entscheidung revidieren wollen. Entscheidend für den Begriff ko-aktiv ist deshalb, dass die Schreibfunktionen während der Nutzungszeit durch die Nutzer festgelegt werden können und nicht während der Entwicklungszeit durch die Entwickler oder bei der Installation eines Systems durch die Administratoren festgelegt und damit für die Nutzer vorgeschrieben werden.

Die Gestaltungsdimensionen sollen helfen, diese Unterschiede transparent zu machen. Ein Wiki beispielsweise eröffnet einen großen geteilten Handlungs- und Wahrnehmungsraum zwischen allen, die schreiben und lesen, führt aber auch dazu, dass durch die fest eingebaute Versionsfunktion alle Fehler und Revisionen einer Person sichtbar sind und nachvollziehbar bleiben. Es gibt Situationen, in denen dies zu Ablehnung des Einsatzes von Wikis führt, weil beispielsweise darüber auch personenbezogene Leistungsmerkmale erfasst und ausgewertet werden können. Das mag in einer Wikipedia möglicherweise für die Nutzer ohne Bedeutung sein. In einem Unternehmen dagegen kann es schnell zu Unsicherheit und Ablehnung führen. Versionsmanagementsysteme vermeiden solche Konstellationen, da sie eine Segmentierung des Handlungs- und Wahrnehmungsraums gestatten, indem Teile eines Textes lokal/individuell bearbeitet und dann durch die Freigabe anderen Beteiligten zugänglich gemacht werden können. D. h. bei der Gestaltungsdimension, bei der die verteilt erstellten und zu verwaltenden Daten der eigentliche Inhalt sind, kommt es darauf an, ob und wie der Handlungs- und 
Wahrnehmungsraum segmentiert werden kann. Das bietet die Möglichkeit, auch an die Segmente Zugriffsberechtigungen so zu erteilen, dass beispielsweise alle Bereiche einsehbar sind, aber nur bestimmte verändert werden dürfen. Hier setzt auch eine weitere wichtige Qualität einer Gestaltungsdimension an, die darauf abzielt festzulegen, welcher Grad an ko-aktiver Bearbeitung der jeweiligen Daten besteht. Zugriffsrechte können z. B. so gehandhabt werden, dass sie mit der Einrichtung des Systems festgesetzt und danach nicht mehr verändert werden können. Oder das System verwaltet die Daten dergestalt, dass im Prozess der Nutzung Zugriffrechte vergeben oder auch wieder entzogen, neue Zugriffsrechte definiert (eine Datei ist sichtbar, kann aber nicht geöffnet werden) oder auch Rechte an andere weitergegeben werden können. Fasst man alle Gestaltungsdimensionen kreisförmig zusammen und unterteilt die Achsen nach dem Grad an ko-aktiver Bearbeitung, der für jede Dimension ermöglicht wird, so erhält man ein Netzdiagramm (Abb. 2), das sowohl für die Analyse als auch zum systematischen Ausloten von ko-aktiven Gestaltungsmöglichkeiten genutzt werden kann.

Zusammenfassend kann festgehalten werden, dass in technischer Hinsicht eine Gestaltungsdimension gekennzeichnet ist durch einen eigenständigen Satz persistenter Daten, der von einem technischen System explizit verwaltet wird, sowie Funktionen zu Erhebung, Verwaltung und Auswertung dieser Daten, die ko-aktiv genutzt werden können.

Das Netzdiagramm mit den Gestaltungsdimensionen für die ko-aktive Innenarchitektur virtueller Wissensräume ist ein Konstrukt, um im Rahmen einer hypothesengeleiteten Gestaltung

- Erfahrungen über frühere Entwicklungsprozesse aufzunehmen und zu reflektieren,

- im Rahmen aktueller Entwicklungsprozesse Anforderungen systematisch hinsichtlich ihrer Wirkungen und Potenziale mit früheren Erfahrungen in Beziehung zu setzen und

- die Eignung einzelner Systeme oder Systemklassen für typische Aufgabenfelder zu identifizieren und zu spezifizieren.

Der Blick auf die Gestaltungsdimensionen erlaubt eine möglichst saubere Trennung zwischen Nutzungskonstellationen und Systemarchitekturen oder Systemklassen, weil Wert darauf gelegt wird, dass technische Eigenschaften und Merkmale unabhängig von der Nutzungskonstellation betrachtet werden können. Die Verwendung des Begriffs ko-aktiv statt kooperativ oder kommunikativ soll genau diese Unterscheidung deutlich 


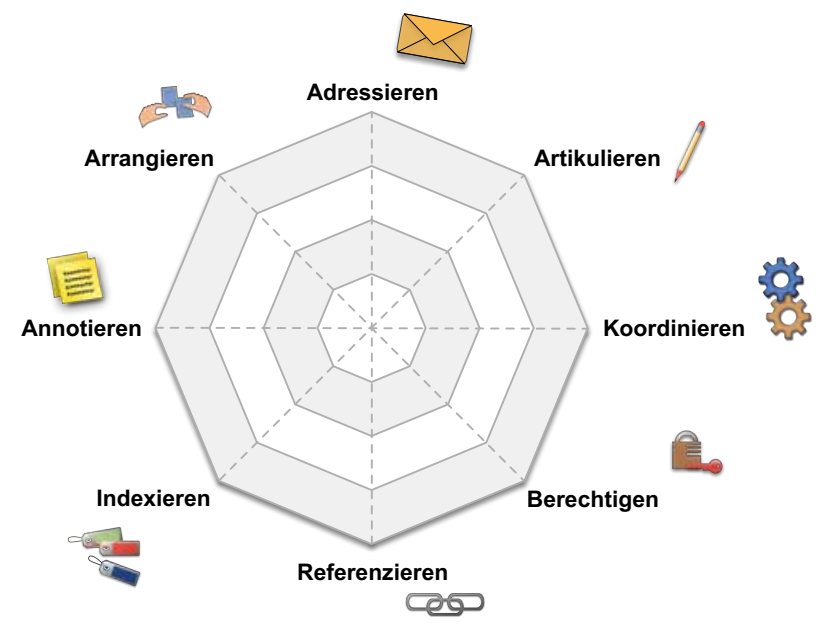

Abbildung 2: Netzdiagramm zur Untersuchung der ko-aktiven Innenarchitektur virtueller Wissensräume

machen, denn das Bereitstellen einer technischen Schreibfunktion, mit der Nutzer z. B. Berechtigungen im laufenden Arbeitsprozess erteilen oder ändern können, sagt noch nichts darüber aus, ob dies im Nutzungskontext in eine Form der Arbeitsteilung eingebettet ist oder ob es zur Unterstützung eines kooperativen oder gar kollaborativen Teams genutzt wird. ${ }^{33}$

Erste Erprobungen im Rahmen von Lehrveranstaltungen zeigten, dass die ko-aktiven Gestaltungsdimensionen zunächst eine Begriffsebene bereitstellen, um die Merkmale technischer Systeme unabhängig von den vielfältigen, heterogenen und oft auch widersprüchlichen Fachausdrücken und Charakterisierungen, mit denen sie beschrieben oder beworben werden, zu beurteilen. So lässt sich z. B. feststellen, dass bei bestimmten Online-Spielen mit vielen weltweit verteilten Nutzern (MMORPG) die Kooperationsunterstützung trotz der Spielszenerie als einem gemeinsamen Handlungsund Wahrnehmungsraum außerhalb des jeweiligen Systems stattfindet, weil die Spieleplattform die jeweils benötigten Gestaltungsdimensionen nicht berücksichtigt.

33 Die Begriffe werden in der Literatur sehr unterschiedlich, zum Teil auch synonym verwendet. Im angloamerikanischen Sprachgebrauch verweist cooperation eher auf eine Form der Arbeitsteilung, während collaboration stärker auf das Setzen gemeinsamer Ziele und die gemeinsame Wissenskonstruktion fokussiert. Vgl. Gabi Reinmann-Rothmeier und Heinz Mandl, Analyse und Förderung kooperativen Lernens in netzbasierten Umgebungen, in: Zeitschrift für Entwicklungspsychologie und Pädagogische Psychologie 34 (2002), S. 44-57, hier S. 45. 
Auf der anderen Seite zeigte sich bei der Untersuchung von Systemen, dass bestimmte Aspekte nicht im Netzdiagramm abbildbar waren. Nach einer vertiefenden Analyse wurde festgestellt, dass eine weitere bisher noch nicht berücksichtigte Gestaltungsdimension vorlag, sodass das Netzdiagramm entsprechend erweitert werden musste. Ein Beispiel hierfür ist die Dimension des räumlichen Arrangierens, die in aktuellen Systemen zunehmend an Bedeutung gewinnt. ${ }^{34}$

Insofern verkörpern die Gestaltungsdimensionen auch jeweils einen Erkenntnisstand, der aber durch neue Einsichten und technische Entwicklungen systematisch weiterentwickelt werden kann. Im nächsten Abschnitt geht es darum zu zeigen, dass die bislang vorgestellten Überlegungen zur Innenarchitektur virtueller Räume auch in der Musikwissenschaft genutzt werden können, um Anforderungen und Möglichkeiten zur Unterstützung der Editionsarbeit zu analysieren und zu präzisieren.

\section{Identifizierung von Unterstützungsfunktionen für die Musikwissenschaft}

Für die Entwicklung eines Ansatzes zur hypothesengeleiteten Technikgestaltung ist es erforderlich, Anforderungen nicht nur als situative Vorgaben für die aktuelle Entwicklung eines Systems zu betrachten, sondern sie in einen größeren Zusammenhang zu stellen, der es ermöglicht, innovative Entwicklung mit allgemein beobachtbaren und geforderten Nutzungsszenarien in Beziehung zu setzen. Dies ist deshalb wichtig, weil man bei der Entwicklung eines Systems meist nicht - wie im vorliegenden Fall auf einen ausgewiesenen Editor historisch kritischer Werkausgaben mit einer besonderen Kompetenz für digitale Editionspraktiken zurückgreifen kann. Anhand einiger Publikationen von Joachim Veit sollen deshalb zunächst einige essentielle Aspekte ko-aktiver Arbeit destilliert werden, die in den Publikationen selbst nicht immer explizit als Anforderungen deklariert worden sind ${ }^{35}$ Mal handelt es sich um Potenziale, mal um Defizite oder Probleme editorischer Arbeit, mal um provokante Aussagen zum Verhältnis analoger und digitaler Editionen oder um Vergleiche verschiedener Praktiken.

Vorab sollte jedoch noch angemerkt werden, dass es im Rahmen dieses Beitrags weder um die Vollständigkeit noch um die Detailliertheit der einzelnen Punkte geht, die hier

34 Vgl. Winkelnkemper/Oberhoff, WebArena (wie Anm. 13).

35 Es muss an dieser Stelle betont werden, dass die ausgewiesene Kompetenz von Joachim Veit für Probleme digitaler Musikeditionen dieses Vorgehen enorm erleichtert. Berücksichtigt wurden dabei die Veröffentlichungen Joachim Veit, Musikedition 2.0: Das ,Aus ' für den Edierten Notentext?, in: editio 29 (2015), Druck in Vorbereitung; Joachim Veit, Noten jenseits des Papiers. Zur Entwicklung von Werkzeugen für die Digital Humanities im Bereich der Musikedition, in: Forschungs-Forum Paderborn 18 (2015), S. 4048; Veit, Es bleibt nichts, wie es war (wie Anm. 4). 
vorgestellt werden, zumal es sich dabei nicht um Äußerungen des Editors handelt, sondern um Destillationen des Autors dieses Beitrags mit dem Ziel, exemplarisch die Möglichkeiten und Probleme einer ko-aktiven Unterstützung zu verdeutlichen. Die jeweiligen Ergebnisse sind naturgemäß nicht fertige Lösungsvorschläge, sondern Hinweise darauf, was bei der weiteren Entwicklung zu beachten ist.

Folgende Punkte sind aus den einzelnen Publikationen unsystematisch herausgezogen worden:

- Umständliche verbale Beschreibung musikalischer Sachverhalte,

- getrennte Darstellung von Notenbild und verbalen Anmerkungen,

- Konsistenz einer Gesamtedition, die sich auf Teileditionen verteilt und sich über verschiedene Bände und verschiedene Seiten eines Bandes erstreckt, die zu unterschiedlichen Zeitpunkten entstehen,

- Nachvollziehbarkeit editorischer Entscheidungen für die Leser,

- unzureichende Prozessierbarkeit von Varianten aufgrund fehlender Objektgranularität,

- taktbasierte Synopse von Quellen; keine Zusammenfassung der Einzelstimmen zu einer Partitur,

- Referenzieren der Takte über die Erläuterungen,

- Ein- und Ausblenden von Zusatzinformationen wie z. B. Taktzahlen,

- Zuschalten von Schichten mit unterschiedlichen Informationen, die sich auf denselben Gegenstand beziehen,

- Bezug auf andere Quellen und Dokumente durch standardisierte Dateiformate und Kodierungen, Verknüpfung von Kontextmaterialien,

- nicht nur textuell vermittelte Genese erfassen, sondern akustische Seite mit einbeziehen,

- halbautomatische Segmentierung des zeitlichen Verlaufs von Musik,

- Auflösen von Gleichzeitigkeit (Einzelstimmen),

- Hervorheben von Einzelstimmen,

- Bewegtbild (Video) zur Visualisierung von Änderungen. 
Bei diesen Punkten fällt zunächst auf, dass sie sich auf eine Fülle unterschiedlicher Aspekte beziehen, die von Fragen der Visualisierung, der Codierung, der Speicherung, der digitalen Verarbeitung, der Objektgranularität etc. beziehen. Die Frage der ko-aktiven Zusammenarbeit ist nur implizit angesprochen und tritt aus einer Informatikperspektive erst auf den zweiten Blick ins Bewusstsein. So ist beispielsweise die Frage nach einer Gesamtedition im Kontext historisch kritischer Editionen immer mit einem mehrjährigen Forschungshorizont verbunden. Auch die bewusst provokant gestellte Frage, ob die neuen Möglichkeiten digitaler Medien „das ,Aus' für den edierten Notentext?" ${ }^{{ }^{36}}$ bedeuten, zielt nicht nur auf die Fragen einer veränderten epistemologischen Perspektive auf den Umgang mit Urtexten und Gesamtausgaben, sondern verweist durch den vorangestellten Begriff „Musikedition 2.0“ darauf, dass der traditionell individuelle, analoge Arbeitsplatz des Editors sich zunehmend ins Netz verlagert und damit die Produkte und Teilprodukte seines Schaffens ebenso wie die zugrunde liegenden Werke und Teilwerke nicht mehr als individualisierte Objekte oder Dokumente aufgefasst werden können, die der alleinigen Rezeption, Transformation und Urheberschaft der jeweiligen Editoren unterliegen.

Doch nicht nur die kritische Reflexion der mit den Zeithorizonten verbundenen epistemologischen Probleme einer konsistenten, den jeweils erreichten Wissensfortschritt einbeziehenden Gesamtausgabe lassen die Notwendigkeit ko-aktiver Bearbeitungsfunktionen erahnen, sondern auch die spezifischen Fragestellungen, die angesichts der neuen Potenziale zunehmend eine Einbettung philologischer Alltagspraxis in einen übergreifenden Forschungsdiskurs bedingen. Dies beginnt bei der Frage nach der Identität bzw. Identifizierung historischer Personen, Orte und Ereignisse und geht über interdisziplinäre Probleme der historisch adäquaten Interpretation unterschiedlicher Fassungen bis hin zur Frage der multimedialen Verknüpfung von Musik und Text. Die im digitalen Medium vergleichsweise einfach herzustellende Verfügbarkeit von Varianten gestattet es Lesern ebenso wie Musikern, Entscheidungen nicht nur zum Editionszeitpunkt festzulegen, sondern zum Aufführungszeitpunkt. Damit ergibt sich aber ein Kreislauf, in dem die vielfältigen Interessen unterschiedlicher Nutzergruppen selbst wieder auf die Arbeit des Editors zurückverweisen. Prozessierbarkeit und Objektgranulariät sind nun nicht mehr allein Fragen der editorischen Bearbeitung, sondern es gilt die Anschlussfähigkeit zu sichern.

Genau hier kann die ko-aktive Innenarchitektur helfen, Fragen systematisch zu stellen, indem zunächst festgestellt wird, welche Gestaltungsdimensionen für welche Interessengruppen und Zeithorizonte notwendig und auch geeignet sind. So kann es wünschenswert sein, Anmerkungen eines Editors von den Anmerkungen eines

36 Veit, Musikedition 2.0 (wie Anm. 35). 
Lesers oder Dirigenten zu unterscheiden. Dabei geht es sowohl um Fragen der Authentizität und Autorenschaft als auch um die Frage, in welchem Zeithorizont und Arbeitsszenario man sich bewegt. In Bezug auf eine abgeschlossene Edition wird hier die Gestaltungsdimension Kommentieren reichen, weil man die Anmerkungen des Editors als Inhalt betrachten kann (Dimension Artikulieren), der von Lesern selbst wiederum nicht verändert werden kann, um die Urheberschaft zu sichern oder um sich vor ungewollten Veränderungen zu schützen. Sollen aber die Anmerkungen selbst zum Gegenstand des ko-aktiven Forschungsdiskurses avancieren, muss man sich zum einen überlegen, welche weiteren Gestaltungsdimensionen hier zu berücksichtigen sind (z. B. Berechtigen, Indexieren, Referenzieren etc.) und wie sie über die Segmentierung der Dimensionen die Gestaltung unterschiedlicher Handlungs- und Wahrnehmungsräume ermöglichen. Zum anderen gilt es zu überlegen, ob und wenn ja, in welcher Form diese ko-aktiven Handlungen über einen längeren Zeitraum oder aber über technisch bedingte Systemgrenzen hinweg kodiert und abgebildet werden sollen, was jeweils erfordert, einen eigenständigen Datenraum für diese Zwecke anzulegen und damit eine neue Gestaltungsdimension zu eröffnen.

Die damit zusammenhängenden Konsequenzen und technischen Fragen sind zum gegenwärtigen Zeitpunkt ebenso wenig befriedigend zu beantworten wie die Fülle der noch editorisch zu bewältigenden Probleme und der sich zukünftig entwickelnden Editionspraxen. Angesichts der komplexen Gemengelage ist es für eine längerfristige interdisziplinäre Forschung notwendig, auch aus der Informatik heraus eine Sprachebene und methodische Hilfsmittel zu entwickeln, die es gestatten, für die ko-aktive Nutzung digitaler Medien den potenziellen Gestaltungsraum zu beschreiben, Potenziale und Konsequenzen frühzeitig zu erkennen, Wirkungen zu systematisieren und die Frage der Einbettung ko-aktiver Funktionen in soziale Praktiken so zu thematisieren, dass eine wechselseitige Befruchtung stattfinden kann, um auch zukünftig im Forschungsfeld der Digital Humanities international federführend agieren zu können.

\section{Ausblick}

Der Forschungsdiskurs zwischen Musikwissenschaft und Informatik hat gerade erst begonnen. Epistemologische Fragen betreffen dabei nicht nur verschiedene Ansätze der automatisierten Erkennung von Musikstücken oder der Modellierung akustischer Strukturen in der Musik, die dem Bereich des distant reading zugeordnet werden können, sondern auch die Unterstützung philologischer Arbeitsprozesse im Bereich historisch kritischer Musikeditionen. Die verschiedenen digitalen Werkzeuge wurden 
hier vereinfacht unter dem Begriff der virtuellen Forschungsumgebung zusammengefasst.

Die Gestaltung virtueller Forschungsumgebungen stellt enorme Herausforderungen dar, die nur interdisziplinär zu bewältigen sind. Dabei wurde deutlich, dass auch in diesem Feld grundlegende epistemologische Fragen angesprochen und bearbeitet werden müssen. Für eine langfristige Zusammenarbeit zwischen Musikwissenschaft und Informatik ist es erforderlich, solche Problemstellungen zu thematisieren und ihre wechselseitigen Bezüge zu analysieren. Dazu ist aber auch auf der Seite der Informatik ein Ansatz erforderlich, der einerseits zu den Diskursen der Digital Humanities im Allgemeinen und der Musikwissenschaft im Speziellen anschlussfähig ist und andererseits gestattet, Gestaltungswissen für virtuelle Forschungsumgebungen zu kodifizieren, zu erweitern und zu aggregieren. Auch wenn sich Gestaltungsprozesse einem formalen Zugang naturgemäß widersetzen, können trotzdem vielfache Unterstützungspotenziale über den Begriff der Wissensarbeit identifiziert, aus anderen Bereichen übertragen und zu einem Ansatz für eine hypothesengeleitete Technikgestaltung verdichtet werden.

Die hier vorgestellten Gestaltungsdimensionen verkörpern ein Gestaltungsrepertoire für die Innenarchitektur virtueller Wissensräume, um speziell den Aspekt der ko-aktiven Nutzung virtueller Forschungsumgebungen zu thematisieren. Dabei wurde gezeigt, dass sowohl Fragen der Objektgranularität und der Segmentierung von Handlungs- und Wahrnehmungsräumen als Grundlage für die verteilte Wissensarbeit als auch epistemologisch bedeutsame Fragen beispielsweise nach der Inhaltsneutralität technischer Werkzeuge und ko-aktiver Funktionen produktiv bearbeitet und wechselseitig aufeinander bezogen werden können.

Das vorgestellte Netzdiagramm als analytisches und konstruktives Hilfsmittel für eine hypothesengeleitete Gestaltung muss dabei selbst noch als Gegenstand der Hypothesenbildung angesehen werden, das in weiteren Forschungsdiskursen erprobt, angepasst und verfeinert werden muss. Dazu gehört auch die Verknüpfung mit anderen Aspekten der Gestaltung solcher Forschungsumgebungen.

Der vorgestellte Ansatz zielt darauf ab, durch das Konzept der möglichst weitgehenden inhaltsneutralen Unterstützungsfunktionen, den Handlungs- und Interpretationsspielraum für die philologische bzw. kulturwissenschaftliche Forschungspraxis zu erhalten und zu erweitern, ohne dabei die Praxis der Nutzer epistemologisch zu beeinflussen oder gar einzuschränken. Zugleich lenkt er den Blick auf technische Gestaltungspotenziale, deren Ausnutzung im nationalen und internationalen Forschungswettbewerb einen Vorsprung ermöglichen könnte. 
Im Sinne dieser Vorgehensweise bietet es sich zwar an, von einer dienenden Funktion der Technik zu sprechen, doch wäre es fatal und verkürzt, die dienende Funktion als eine zu verstehen, bei der es nur darum geht, technische Systeme nach festen Vorgaben zu implementieren. Um auf Dauer wirklich dienende Funktionen zu konzipieren und sie nachhaltig und alltagstauglich umzusetzen, ist der interdisziplinäre Diskurs zwischen der Informatik und den Geistes- und Kulturwissenschaften unverzichtbar. Für den Erfolg dieser Zusammenarbeit ist jedoch weniger der spezielle hier vorgestellte Ansatz entscheidend, sondern die Frage, ob überhaupt der Ansatz einer hypothesengeleiteten Technikgestaltung mit den ihnen zugrunde liegenden Annahmen, damit verbundenen epistemologischen Fragestellungen und den technischen Gestaltungsperspektiven eine geeignete Grundlage für den interdisziplinären Diskurs abgibt. Anders ausgedrückt: Der Ansatz einer hypothesengeleiteten Technikgestaltung verkörpert selbst eine Hypothese im Forschungsdiskurs zwischen der Musikwissenschaft bzw. den Digital Humanities und der Informatik. 